\title{
Une esthétique du fétiche
}

An Aesthetics of Fetishes (Bwaba, Burkina Faso)

\section{Michèle Coquet}

\section{OpenEdition}

Journals

Édition électronique

URL : http://journals.openedition.org/span/1034

DOI : $10.4000 /$ span. 1034

ISSN : 2268-1558

\section{Éditeur}

École pratique des hautes études. Sciences humaines

\section{Édition imprimée}

Date de publication : 1 septembre 1987

Pagination : 111-140

ISSN : 0294-7080

\section{Référence électronique}

Michèle Coquet, « Une esthétique du fétiche », Systèmes de pensée en Afrique noire [En ligne], 8 | 1987, mis en ligne le 16 octobre 2013, consulté le 30 avril 2019. URL : http://journals.openedition.org/ span/1034; DOI : 10.4000/span.1034 


\title{
ONE ESTHETIQUE DU FETICHE
}

\author{
par
}

\section{Michèle Coquet}

Les sociétés africaines fabriquent pour leur usage quotidien ou religieux beaucoup d'objets qui atteignent une grande perfection technique; cependant, il en existe d'autres qui nous apparaissent peu aboutis dans leur forme et de facture sommaire. Ces objets sont souvent en matériaux périssables et sont en général entourés d'une très grande attention; ceux que l'on dénomme couramment objets-fétiches font partie de cet ensemble. Nous nous proposons de prendre un de ceux-ci en considération et de voir quelles sont les corrélations qui se mettent en place entre la structure de cet objet et la manière dont le groupe propriétaire et utilisateur se pense par rapport à lui dans son histoire et son organisation particulière. I'un et l'autre évoluent en parallèle sans qu'il soit possible de dire si l'objet, par sa composition interne, est une illustration, une projection matérielle, entièrement soumise à un système d'interprétations sociale ou mythologique. En effet, l'objet est toujours en partie indépendant dans sa forme: il peut très bien être utilisé par une autre société que celle au sein de laquelle il a été créé, en étant le support d'interprétations différentes, mais la manière dont il est construit influe sur l'ensemble des concepts qui s'y rapportent. Dans le travail que nous présentons ici, l'hypothèse de cette autonomie de l'objet ne sera pas développée; cependant, abordant l'objet du rituel d'un point de vue morphologique, il nous semble important de préciser ce fait, déjà relevé par d'autres auteurs, 
comme Franz Boas. Celui-ci cite dans Primitive Art (F. Boas, 1955: 124) le cas d'un sac indien en tissu, orné de motifs géométriques, acheté par lui à une femme indienne de Colombie Britannique, qui l'avait elle-même obtenu d'une autre tribu; ne connaissant pas la signification des dessins, la femme avait réinterprété l'ensemble selon le mode propre à la tribu à laquelle elle appartenait, et pour en renforcer la cohésion, elle avait elle-même ajouté d'autres signes. Boas n'a pas tiré toutes les conclusions de cet exemple : si l'autonomie de l'objet apparất bien, il manque par contre les éléments nécessaires pour retrouver la logique interprétative induite par l'organisation des graphismes eux-mêmes.

Après avoir considéré un objet-fétiche tel qu'il est pensé dans la société que nous étudions, quelques propositions esthétiques seront avancées à son propos, tentant de le définir au-delà (ou en-deçà) de la signification religieuse et mythique, par l'appréhension de sa forme et de sa matière.

Nous avons pris notre exemple chez les Bwaba du Burkina Faso, de la région de Houndé. Ceux-ci sont connus pour leur sens de la collectivité et leurs aspirations égalitaires ${ }^{1}$ : une religion communautaire, le culte de Doo, très vivante, donne sa cohésion à une société par ailleurs est fragmentée si l'on considère le nombre de ses dialectes et la répartition territoriale clairsemée des villages. Dans ce contexte, les cultes fétichistes n'ont pas, semblet-il, eu l'occasion de beaucoup se développer : peut-être est-ce dû au fait que leur existence est souvent liée à la formation d'associations d'individus, de collèges se réclamant du culte d'un fétiche

\footnotetext{
${ }^{1}$ La société bwanu est formée de trois groupes socio-économiques distincts, paysans, artisans forgerons et griots ; souvent musiciens et tisserands, les forgerons exercent la fonction normalement réservés aux griots, ces derniers étant rejetés par les paysans. Dans le sud du pays bwanu, paysans et forgerons forment le noeud de la communauté (cf. Coquet, 1986: 135, note 1). Ces deux groupes sont en principe endogames. La parenté est patrilinéaire : J. Capron l'organise en groupes agnatiques et en lignées. Nous préférons pour notre part adopter la terminologie traditionnelle : nous parlerons donc d'ensembles de lignages formant un clan, c'est-à-dire se réclamant d'un ancêtre mythique commun et d'un même interdit totémique, chaque lignage se référant à une généalogie précise d'ancêtres connus.
} 
particulier, pouvant rompre cet idéal communautaire par I'importance accordée en ce cas à un lieu (celui où se trouve le fétiche) et à un groupe (celui qui le possède). Si nous faisons abstraction des amulettes, talismans, bracelets et autres petits objets qui forment le bagage élémentaire de tous les Bwaba et qui sont d'usage individuel, il reste à notre connaissance peu d'objets-fétiches. Mais encore faudrait-il savoir ce qui répond à cette dénomination et les critères de sélection choisis.

\section{Le monde des objets}

Pour les Bwaba, tout objet occupant une place prédominante dans un rituel, ou donnant lieu à un culte particulier, peut recevoir l'appellation de "fétiche"; parmi ces objets, nous en choisirons un qui semble correspondre plus particulièrement à une certaine conception du "fétiche", telle qu'elle apparất dans les ouvrages consacrés à l'Afrique. L'intérêt pour le fétichisme ayant donné lieu à des listes d'objets où se mêlent indifféremment statuettes, talismans, amulettes, etc., nous tenterons de voir ce qui unit cet ensemble de matériaux dissemblables. Pour cela, nous partirons de l'objet conçu comme artefact, chose fabriquée, sans tenir compte des croyances qui y sont liées.

D'après leur position dans l'espace, nous voyons que les objets-fétiches appartiennent au mobilier, dans le sens où l'utilise A. Leroi-Gourhan lorsqu'il parle d'art mobilier, ce qui comprend tous les objets figurant dans, ou aux alentours, d'une habitation. Ils forment un groupe hétéroclite d'éléments mobiles, virtuellement ou réellement, par opposition à ceux qui sont fixes - nous pensons plus particulièrement chez les Bwaba aux autels d'ancêtres, autels de brousse, autels de doo - qui, une fois construits en un lieu, y demeurent solidement implantés et comme ancrés au sol qui les porte. La mobilité, réelle ou virtuelle, implique que ces objets sont susceptibles d'être changés de place. Selon l'emploi qui en est fait, ils seront d'abord cachés puis exhibés à certains moments 
de leur manipulation, demeureront cachés en permanence ou seront au contraire ostensiblement montrés. Ces différentes possibilités permettent de les distinguer des autels fixes, qui, par leur nature propre, et même si l'accès peut en être réglementé et codifié, ne présentent pas de semblables variabilités. Parmi les objets "fétiches" de ce genre, nous pensons aux statuettes nkonde, ou nkisi, plus communément appelées "fétiches à clous", de provenance congolaise, qui sont sorties de leur abri à certaines occasions et exposées à la pleine lumière du soleil pour être "revitalisées".

Ces objets, plus ou moins mobiles, hésitant entre l'état caché et l'état montré, évoluent dans ce que nous pouvons appeler la sphère de I'humain : en effet, ils paraissent, d'une certaine manière, être attachés à la personne humaine, soit que celle-ci les porte sur elle, soit qu'ils occupent des espaces parmi les plus humanisés que fréquente et qu'habite l'homme, c'est-à-dire la maison, le village, ou sa périphérie immédiate : c'est là qu'ils sont de préférence entreposés, et non pas dans des endroits plus éloignés tels que les champs ou la brousse.

L'objet-fétiche apparất avant tout comme une "chose" du village. Que cet objet soit d'usage collectif ou individuel, comme les talismans ou les amulettes, ce qui nous semble important, c'est sa situation spatiale, être près du corps ${ }^{2}$ - sur le corps de la personne elle-même ou dans les maisons -, par comparaison avec les autels que nous avons appelés fixes, qui peuvent éventuellement se trouver éloignés du village - autels de brousse, doo de brousse chez les Bwaba, au pied d'un arbre, dans un bosquet, en plein champ, etc. Nous connaissons d'ailleurs chez les forgerons bwaba le cas de dessins d'objets-fétiches inscrits par scarification dans le corps, ce qui est un exemple du degré possible de fusion qui peut être atteint entre l'objet et l'individu.

\footnotetext{
2Les expressions "sphère de I'humain" et "être près du corps" peuvent rappeler la classification des arts plastiques proposée par M. Mauss en fonction de leur relation au corps. M. Mauss a en effet divisé les différents arts en arts du corps, arts des entours, arts figuratifs autonomes. Ici, c'est avant tout à la notion d'art mobilier que nous nous référons.
} 
Nous en concluons, tout en sachant que ce découpage demeure d'une précision relative (nous l'avons mis en place en fonction de critères de manipulation et de positionnement spatial), que le type d'autel auquel nous nous intéressons peut se définir à partir des particularités suivantes : il est plutôt mobile (transportable) que fixe, et situé dans une zone assez délicate à déterminer, mais que nous avons observée comme étant près du corps. De plus, il est de nature complexe, étant composé ordinairement d'éléments hétérogènes. Ce dernier point n'est pas sans rappeler un sens particulier du mot "fétiche" cité par C. Ratton, concernant "l'or fétiche" :

"L'or que ceux de Dinkira nous apportent est bon et pur, excepté qu'ils y mêlent trop de fétiches, qui sont une espèce d'or composé de toute sorte d'or mis en oeuvres et dont quelques-unes ont une plaisante figure" (Ratton, 1951) 3.

Après cette première tentative de définition des traits particuliers à l'objet-fétiche le différenciant des autres objets du rituel, nous nous proposons de voir en quoi l'idée de l'existence d'une "force" peut être utile pour comprendre l'objet dans sa matérialité et dans sa forme.

Une des propriétés de l'objet-fétiche fréquemment invoquée par les textes ethnographiques serait qu'il est le support d'une "force", ou d'une "puissance", bénéfique (protection) ou maléfique (sorcellerie). Suivant la démarche comparative qui est la nôtre - un objet rituel parmi d'autres -, il n'est pas certain que s'appuyer sur cette proposition de l'existence d'une force soit satisfaisante. Contrairement à d'autres qui n'ont de sens que le temps du rituel, comme ces pierres placées dans les branches des arbres pour retarder le coucher du soleil et qui n'ont "de signification mystique que pendant la durée du rite" (Evans-Pritchard, 1971: 106), la consécration de l'objetfétiche semble être faite une fois pour toutes, consécration au cours de laquelle il devient le lieu de résidence privilégié d'une "force" particulière. Cette idée de force caractérisant l'objet-fétiche

3Le propos rapporté par C. Ratton est celui d'un voyageur, Bosman, qui a écrit une "Description de la Guinèe" (Utrecht, 1704). 
est reprise par de nombreux commentateurs : ainsi, Jean Laude sur les nkisi congolais (Laude, 1979: 125) :

"Les fétiches naturels* doivent leur vertu magique aux forces qui les habitent et leur viennent de la nature : coquillages, cailloux, morceaux de bois, excréments, etc. Les fétiches imprégnés* sont des sculptures qui détiennent leur pouvoir des opérations effectuées par un être doué de facultés surnaturelles : le nganga (l'hommemédecine). Les statuettes n'apparaissent ainsi que comme de simples supports, ou, si l'on préfère, des conducteurs de la force magique". *(C'est nous qui soulignons).

Dans ces quelques lignes apparâ̂t cette vision du fétiche comme combinaison d'éléments hétéroclites dont l'association permettrait la concentration d'un plus grand potentiel de "forces" sur le même objet (remarquons que la fonction de la statuette est réduite à celle de simple support). L'auteur continue :

"La sculpture (...) et les matières dont elle est composée ou qui lui sont adjointes sont en elles-mêmes inertes. Elles sont susceptibles de recevoir ou de fixer une force pour un certain laps de temps mais c'est le nganga qui, par des rites appropriés, par la récitation de certaines formules, alerte cette force et l'oriente, dans un sens ou dans un autre : si, enfonçant un clou dans une statuette, le nganga mayombe libère une force, cette force doit être dirigée et contrôlée. C'est le sorcier qui, par une incantation, précisera le rôle bénéfique ou maléfique de son opération" (Laude, 1979:126).

C'est à dessein que nous prenons J. Laude pour exemple. En effet, il résume une certuine conception de l'objet-fétiche : celui-ci est un composé d'éléments hétérogènes, support d'une force, et qui nécessite l'intervention de la parole pour devenir opératoire. L'auteur, bien que spécialiste de l'art primitif et en particulier africain, n'a pas pu se détacher de certains points de vue dorénavant classiques: plus la composition de l'objet est hétéroclite, plus, semble-til, il réunit de "forces", puisqu'à chaque élément est associé une force. Force et parole, parole qui transmet la force, forment un couple indispensable à l'intronisation de tout objet rituel mais lui demeure comme périphérique et ne suffit pas à le définir dans sa structure même. En effet, l'objet existe avant et sans la parole : reprenant la proposition de L. de Heusch (1974: 233), nous pouvons dire que l'objet-fétiche est un instrument, un outil de communication, comme d'autres objets du rituel (couteaux, masques, etc.), mais d'une complexité particulière. Si l'outil sert avant tout à agir sur quelque 
chose, sa conception, puisqu'il doit par sa forme et sa matière correspondre à l'usage auquel il est destiné, répond à des exigences à chaque fois spécifiques. "Un outil humain est (...) un objet fagonné, transformé (souligné par nous) de manière à pouvoir être utilisé commodément et efficacement pour accomplir un certain genre d'action" (définition du Petit Robert). Nous revenons à la définition donnée au début de ce texte : le fétiche est un objet mobilier, un "outil", que l'on porte sur soi ou à proximité en vue d'un usage particulier dont dépendent, en partie seulement, sa forme et sa matière.

\section{Le principe d'hétérogénéité}

La particularité formelle la plus remarquable de l'objet-fétiche, celle qui en tout cas a frappé les observateurs, est la grande hétérogénéité des matériaux qui le composent, souvent agglutinés jusqu'à former une masse compacte. En 1911, P. Guébhard circule dans les villages bwaba et note à propos de ce qu'il nomme le "culte des grigris" ce fait révélateur :

"Les grigris des villages sont les objets les plus hétéroclites du monde. Ils diffèrent essentiellement de forme et de composition dans chaque village. Ce sont quelquefois des amas poussiéreux de choses indéfinissables, cornes de mouton ou d'antilope, vieilles ferrailles, morceaux de bois ou de chiffons, sonnettes, morceaux de calebasses, ficelles, osselets (...). D'autres fois les grigris sont cousus dans un sac en peau de mouton dont le contenu est inconnu" (Guébhard, 1911: 126).

H. Labouret, un peu plus tard, en 1927, recueille auprès d'un informateur de vieilles recettes de fabrication d'objets semblables et établit la liste des éléments "indéfinissables" qui les composent. La recette concerne un korte, ensemble de poudres amalgamées dans une matière grasse, le tout enfermé dans une corne d'animal sauvage ou domestique :

"Pour préparer le korte, va dans la brousse, cherche un vieux caîlcédrat, enlève à ce vieil arbre l'écorce du côté du couchant. Coupe les racines qui traversent le sentier ; cueille des fleurs de (?), prends la salive d'un agonisant, des racines de ntaba koumba (légumineuse), des racines de blantye, creuse pour tirer des racines de chiendent, cherche une tête de vipère. Réunis tout cela, laisse-le sécher, pile-le, réduis-le en poudre; cela 
fait, ajoute une poterie de l'urine d'une vieille femme et du sang de coq rouge à cette poudre, mets cela sur le feu. Au moment où le mélange bouillira, dis : Au nom de Dieu (...), Bala qui ne s'est pas levé, homme caché, que Dieu n'a pas caché, génie qui n'est pas là, dont le nom est là, je t'ai cherché pour faire ton office, faire ton office pour nous" (Labouret, 1927).

R. Lehuard, à propos des statues nkonde (fétiches à clous), dresse une liste similaire (1980: 84) : les ingrédients qui sont contenus dans celles-ci se composent d'argiles blanche et rouge prises dans un cours d'eau, d'éléments végétaux, d'écorce d'arbre, de fragments d'animaux, de becs d'oiseaux et de coqs, etc. Nous arrêterons là la liste des descriptions qui s'accordent toutes sur le caractère composite de l'assemblage qui fait la matière de l'objet. Tant pour le korte que pour les nkonde, il y a un choix d'éléments dont la seule règle d'agencement semble être à première vue celle de rassembler des matières appartenant aux différentes catégories d'éléments naturels (animal, végétal, minéral et humain). Ces éléments sont associés à une compétence particulière : ainsi, par analogie, la plante piquante manifestera la valeur défensive, les crocs de lion, la puissance, les becs d'oiseaux, la rapidité. L'organisation de l'objet-fétiche obéit dans ce sens à la logique concrète : des éléments provenant de différentes espèces sont choisis, investis de signification, mis en forme, organisés logiquement en vue de la création d'une oeuvre plastique exprimant une certaine relation de l'homme au monde. Ainsi, si l'on peut penser qu'il existe bien une mise en place d'équivalences primaires, de type analogique, - crocs de lion = force, caractère piquant d'une plante $=$ défense, plume d'aigle = puissarce, etc. - , rien ne nous permet de dire exactement pourquoi un élément naturel est considéré plus prégnant qu'un autre, même si la pensée symbolique s'y emploie avec ardeur. S'appuyer sur le principe de la logique concrète pour comprendre I'objet-fétiche tend donc à réduire la plastique de l'objet à un ensemble d'éléments réunis par une opération de transcodage des données du monde sensible - la puissance du lion à travers ses crocs - en autant d'unités symboliques ; or l'analyse plastique de l'objet (matière, forme, couleur) ne doit pas être négligée. 
En conclusion, nous dirons que l'effet d'hétérogénéité est obtenu, dans la plupart des cas, matériellement, par le caractère composite des éléments inclus, amalgamés, par addition d'unités morphologiques spécifiques, hétérogénéité qui est présente aussi dans la somme des programmes de fabrication, allant de la recherche, de la cueillette des éléments jusqu'à leur manipulation et leur transformation définitives. Tout objet fini, outil ou oeuvre d'art, implique la succession de ces programmes de fabrication ; la particularité de l'objet "fétiche" est, nous le verrons, de rendre souvent manifeste, dans la plastique même de l'objet, la succession de ces phases. Si cette succession n'est pas manifeste, elle est présupposée comme dans le cas du korte, car toutes les opérations nécessaires sont mémorisées et donnent de la valeur à l'objet : les traces de fabrication participent de l'esthétique fétiche.

\section{L'objet-fétiche "nazii"}

Nous avons défini l'objet-fétiche comme un objet mobilier, donc transportable, situé dans l'espace interne des maisons, composé d'éléments hétérogènes. Un tel objet existe chez les Bwaba de la région de Houndé : il s'inscrit dans le cadre d'un culte clanique puisqu'il concerne une divinité totémique commune à un ensemble de lignages, le culte lui-même étant pratiqué de manière autonome au sein de chaque lignage.

Chez les forgerons, le plus âgé du clan est chargé de garder cet objet : il est déposé à l'intérieur de la maison des ancêtres du lignage, reconnu comme étant généalogiquement le plus proche du groupe originel. Dans cette maison se trouve tout le matériel cultuel se rapportant à la fondation du clan, matériel qui, lorsque le lignage propriétaire disparast faute d'héritier, peut être amené $\grave{a}$ changer de territoire, c'est-à-dire souvent de village, pour 
devenir la propriété d'un autre lignage 4. J. Capron décrit un objet semblable nommé dans les régions septentrionales nadû ; l'étymologie proposée par l'auteur décompose le terme en de dâ, la "queue", et na, du verbe na/naa, creuser profondément, forer, chercher par tous les moyens, déployer ses efforts pour, faire l'impossible pour... (Capron 1978: 61). Les Bwaba de la région de Houndé classent quant à eux cet objet-fétiche dans la catégorie des objets wahu ; wahu est également utilisé pour les amulettes, talismans, etc., et s'oppose ainsi au terme qui désigne les autels proprement dits, qui est nuu, "tête", comme dans mieba nuu, littéralement "tête des ancêtres" pour l'autel des ancêtres. L'objet wahu que nous avons étudié est celui du clan forgeron didiro ; il est appelé par ses propriétaires le didiro nazii. zii est la "queue", en dialecte de Houndé, comme dâ dans nada. na, par contre, est la vache, soit nazii, "queue de vache". nazii correspond au nazumbwe dont parle J. Capron (1957: 96), qui a la même étymologie.

Si nazii désigne la queue de vache qui est l'instrument rituel indiquant le statut social et religieux de son porteur (chef de terre, prêtre de doo, ou plus simplement chef de lignage), il n'est pas de la même forme que didiro nazii. didiro nazii et nazii, sont dans un rapport d'équivalence symbolique et non pas formelle. Nous reviendrons plus longuement sur l'étymologie de didiro.

L'objet que nous connaissons correspond à la description qu'en fait J. Capron. Paquet de forme vaguement ovoide et de dimensions importantes puisqu'il peut atteindre une soixantaine de centimètres, le didiro - tel est le nom que nous lui donnerons dès lors -, se présente sous la forme de peaux de chiens sacrifiés, enroulées, superposées les unes aux autres jusqu'à faire paquet. Les seuls animaux à pouvoir être immolés en vue de la confection de cet objet sont les chiens à robe fauve, ou, éventuellement, des poules et des chèvres noires.

\footnotetext{
4L'objet dont il est question ici appartient à un clan forgeron: tous les clans forgerons en ont un. Nous n'avons pas pu vérifier s'il en était de même chez les paysans. Au nord du pays bwamu par contre, l'objet, d'après J. Capron, est utilisé par les paysans.
} 
Nous ne nous étendrons pas sur la symbolique des couleurs, déjà connue, mais nous donnerons, à titre indicatif, quelques précisions : la couleur noire a une valeur négative et est associée généralement à ce qui est dangereux, au mal, à la maladie, à la trahison (G. Le Moal, 1983: 29) 5 . Les forgerons eux-mêmes ont cette couleur comme attribut emblématique, puisqu'elle est celle du charbon produit par leur activité. Le rouge est bien entendu la couleur du sang, mais aussi de la vie, de la violence et de la force. Comme le noir, il est pensé comme dangereux. Les deux couleurs s'opposent en cela au blanc, couleur de l'ordre : ainsi, l'organe examiné du poulet sacrifié devra être blanc (le poulet au "coeur clair") si la victime est acceptée par le destinataire de l'offrande ; de même seront blanches la cendre ou la craie offertes sur les autels d'ancêtres. Le didiro ne peut être mis en contact qu'avec ce qui est rouge ou noir et non avec ce qui est blanc.

Le fait qu'il s'agisse exclusivement de sacrifice de chien n'est pas non plus sans importance : le chien est en effet un animal rétif, violent, et difficile à tuer. Comme l'a bien fait remarquer G. Le Moal (1983: 41), il est ambivalent : au village, il est gardien des bêtes et des maisons, en brousse, il est prédateur, tueur d'animaux. Le choix de cet animal reconnu comme agressif, la couleur rouge de son pelage, marquent avec évidence le caractère de "puissance dangereuse" qui est accordé à l'objet par ses propriétaires. Ajoutons que le chien est en général, prescrit comme victime lors d'événements qui présentent pour la communauté villageoise des moments de tension dramatique, comme l'initiation, la mort brusque du chef de terre ou une rupture d'interdit. Il en est de même lorsque ces événements concernent un lignage ou le clan tout entier : ainsi, pour le clan didiro, en cas d'acte sacrilège commis dans une forge ou au noment du rituel de changement du tronc de l'enclume sacrée, en cas de

5 En étudiant les couleurs des victimes sacrificielles chez les Bobo, limitrophes des Bwaba, G. Le Moal note combien la catégorie du noir est complexe : le noir désignerait non seulement le mal ou la maladie, mais aussi "tout ce qui échappe à la connaissance", comme les secrets de l'initiation, l'espace de la brousse, le ciel orageux, etc. 
mort inexpliquée, ou lors du mariage d'un garçon du lignage. En diautres circonstances, semble-t-il mineures (demande de pluie, mariage d'une fille du lignage, acte propitiatoire pour une faute peu importante), ce sont la poule ou la chèvre noire qui sont ordonnées. Si l'événement survient au sein du lignage descendant du groupe fondateur, l'ensemble des chefs de lignages didiro doit se déplacer auprès du didiro nazii de ce lignage héritier.

\section{Le coeur du didiro nazii}

Pour marquer la cohésion des membres du clan, leur parenté et leur appartenance à une même histoire, tous les lignages possèdent un morceau de l'objet original. Chaque lignage didiro a construit, en prenant ce morceau comme noyau, un nouvel objet, de sorte que chaque forge a le sien. Si tous les objets wahu de ce type sont de forme semblable et de matière principale similaire (peaux de chien), la composition centrale varie selon les clans propriétaires : semblables dans ce qu'ils donnent à voir, ils sont différents dans ce qu'ils contiennent: les "racines de l'autel", wahu niina (Capron, 1957: 58)6. Outre les peaux de chien qui font l'enveloppe feuilletée et épaisse, celle qui, en fait, façonne l'objet, lui donne sa forme, il y a certains ingrédients, situés au coeur, qui donnent à l'objet sa spécificité. J. Capron (1957: 58-59) en dresse une longue liste à propos des nadû des régions septentrionales : "ossements de la personne de brousse" qui a transmis l'autel (I'auteur précise: par "personne de brousse", Ies Bwaba désignent une catégorie d'êtres surnaturels, les génies de brousse, qui forment une société semblable, symétrique à celle des hommes, mais dans l'espace de brousse), écorces et racines d'arbres provenant du territoire de brousse en relation avec cette "personne de brousse", ossements et dépouilles d'autres animaux sauvages, représentation sous forme de graines enveloppées dans une bande de cotonnade des huit premières plantes créées par

6Nous retrouvons ici le terme wahu, ce qui nous laisse penser que la dénomination est la même dans les régions septentrionales. 
Dieu (dofini)7 (nous ne connaissons pas cette pratique dans le sud du pays ; il pourrait peut-être s'agir d'une influence dogon), un élément venant de l'autel de brousse du village (tige de graminées sauvages), un élément venant de l'autel de fondation de la communauté, un élément métallique de la forge, etc. La description que nous ferons du didiro nazii sera moins précise que celle-ci, le secret du contenu du paquet étant particulièrement bien gardé.

Didiro est composé, comme les autres fétiches de cette catégorie, de peaux de chiens sacrifiés et c'est bien le noyau qui diffère. A notre connaissance, celui-ci est formé d'éléments animaux (un squelette de poisson), végétaux (des grains de mil rouge) et minéraux (un morceau de mâchefer, résidu de la fonte du métal, ainsi qu'une plaque métallique, qui représente le premier couteau sacrificiel, nommé doo comme l'entité surnaturelle doo dont il est une des manifestations, couteau qui est utilisé soit comme support sacrificiel, soit comme rhombe). Nous avons parlé de squelette de poisson: or, celui-ci est nommé également didiro, ce qui nous amène à expliciter l'étymologie du terme. Plus qu'un patronyme, didiro est une dénomination qui a trait à l'histoire mythique du clan. Elle désigne en premier lieu, dans la langue bwanu, une variété de poisson vivant en eau douce et dont les décharges électriques sont redoutées ${ }^{8}$.

L'épisode de la dernière migration du groupe forgeron est assimilé à l'événement mythique qui l'a constitué en clan proprement dit se réclamant depuis d'une mêre origine. Les forgerons didiro prétendent venir d'un endroit situé à une quarantaine de kilomètres à l'Ouest de leur zone actuelle de dispersion (la région de Houndé).

Les versions que nous avons recueillies de ce mythe concordent avec les informations données par les différents chef's de lignage. Cette homogénéité est d'autant plus remarquable que l'aire de dis-

7Les Bwaba croient en un dieu lointain, dofini, créateur de l'univers et de tous les êtres qui l'habitent. Ils s'adressent rarement directement à dofini, lui préfèrant des intermédiaires tels que doo, l'envoyé de dieu auprès des hommes, ou les ancêtres.

8 Malapterurus electricus, famille des Malapteruridae, appelé parfois silure électrique; poisson de grande taille, jusqu'à un mètre, il a une capacité de décharge électrique de 350 à 450 volts. 
persion est assez vaste (une cinquantaine de kilomètres du nord au sud). Le lieu d'origine, vuhu, est évoqué comme celui de la forge unique, la forge idéale telle qu'elle existait avant l'éclatement du groupe, et donc là où tous étaient rassemblés en un seul village. Après comparaison de ces récits, l'histoire du clan didiro peut être exprimée ainsi.

La nuit où les forgerons au vuhu plantèrent le bois de l'enclume sacrée de la forge en terre, ils furent guettés par un lépreux chargé par les paysans de les surprendre dans l'accomplissement de leurs rituels. Le lépreux fut découvert et sacrifié. Craignant les représailles des paysans, les forgerons s'enfuirent vers l'est. Là, ils furent arrêtés dans leur course par une rivière en crue. Le premier ancêtre Zama9 s'adressa au génie des lieux. C'est didiropoisson qui répondit à l'appel ; pour venir en aide aux fuyards, il mit à contribution un deuxième animal, une tortue aquatique, dempi10. Celle-ci se posa sur le côté de manière à avoir le corps à la verticale, perpendiculaire au niveau de l'eau, et les flots se séparèrent. Les forgerons purent passer sur l'autre rive. didiro dit : "Que ceux qui ne sont pas didiro soient emportés par les eaux". Lorsque les paysans voulurent à leur tour s'avancer, la décharge électrique envoyée par didiro les tua. Les forgerons se dispersèrent à travers les villages bwaba de l'est, bien que certains lignages se trouvent encore à l'ouest du lieu géographique où eut lieu l'événement. Lors de cette traversée, un contrat fut passé entre didiro et le premier ancêtre. didiro apparât comme le donateur de tout le matériel cultuel, celui propre aux forgerons en tant que groupe social déterminé : les cinq masques, qui semblent représenter les cinq ancêtres claniques, ceux-là même qui avaient eu l'initiative de la traversée mythique, et surtout les instruments nécessaires au culte de doo. Lors de la fuite des forgerons, "didiro

\footnotetext{
9Premier ne renvoie pas bien sûr à une généalogie mais plutôt au caractère mythique du début de l'existence du groupe.

10 Tortue aquatique de la famille des Tryonychidae. De caractère agressif, elle peut atteindre jusqu'à 95 centimètres de diamètre. Les tortues de cette famille possèdent un long cou et une tête prolongée par une trompe. La carapace, bien que complètement ossifiée, demeure souple, et est recouverte d'une peau épaisse.
} 
a vomi les couteaux doo", ces couteaux sacrificiels dont nous avons parlé et qui permirent d'effectuer les premiers actes rituels: sectionnement du cordon ombilical pour consacrer la première nais- sance et scarifications. didiro exigea en échange que sa chair ne soit plus consommée et que lui soient faits périodiquement des sacrifices (chien roux ou poulet et chèvre noirs) au marigot où il réside ${ }^{11}$, ou sur l'objet nazii qui fut, d'après le mythe, donné aux forgerons comme marque de l'alliance.

Par ailleurs, didiro semble être une des manifestations possibles à la fois de dieu-dofini et de doo. En vomissant les rhombes, conçus comme des avatars de doo, didiro-dofini engendre doo. Les couteaux réels, que chaque lignage possède, portent inscrits le signe de didiro, une ligne de chevrons représentant le squelette du poisson ; ceci marque à la fois la filiation divine didiro-dofini

doo (doo étant l'envoyé de dofini), et l'origine également divine du clan forgeron, troisième terme de la suite d'attribution par le donateur surnaturel :

\section{didiro-dofini $\longrightarrow$ doo $\longrightarrow$ forgerons}

didiro est aussi appelé "jumeau", doo henbeni, de doo : ceci indique bien que l'identité de didiro est floue, puisqu'il est à la fois assimilé à dofini et pensé comme un double de doo. doo étant l'envoyé de dofini pour l'ensemble des Bwaba, didiro serait plutôt doo réservé à l'usage clanique et lignager (ce qui expliquerait son assimilation à doo). La composition du noyau du didiro nazii n'est pas sans rapport avec celle que décrit G. Le Moal (1980: 125) à propos de l'autel de Dwo chez les Bobo, où I'on trouve également des rhombes et une dépouille de l'animal se rapportant à la révélation première de Dwo à un clan 12 . Dans notre cas, le rôle initiateur et civilisateur de didiro (les forgerons didiro disent qu'il leur a tout donné, même la mort) double par ailleurs celui de doo dans

${ }^{11}$ Chaque lignage didiro a en sa possession un marigot ou une portion de marigot considérés comme le lieu de résidence de didiro.

${ }^{12} \mathrm{La}$ composition de l'autel de doo chez les Bwaba ne comporte pas à notre connaissance de dépouille animale, le terme doo renvoyant à un culte collectif et non clanique. 
d'autres récits qui traitent de l'histoire de doo comme envoyé de dofini : tout porte à croire que didiro est une représentation clanique de doo.

La mort a pour origine le meurtre de didiro-dofini par le premier fils de doo, binluio13. Ce dernier, après avoir été un être surnaturel, est ensuite fait homme : il est conçu comme le premier ancêtre forgeron. Sa tombe se situe sous le bois de l'enclume, appelée binluio lunnuu, "tête binluio de la forge", tête désignant le lieu de l'autel. binluio, en tuant et en consommant le corps de didiro, fait apparaitre la première mort, le premier sacrifice, et, accompagnant ces événements, la condition humaine, puisque binluio lui-même fut le premier humain à mourir. Le signe de ce meurtre primordial est inscrit sur le visage de tous les Bwaba par le dessin des "larmes" qui consiste en deux ou plusieurs traits partant de la racine du nez jusqu'aux pommettes.

En résumé, nous voyons que didiro-objet prend place dans un récit typique dans ce contexte, celui de la constitution d'un clan comportant plusieurs phases : détachement d'un groupe d'individus de l'ensemble de la communauté, déplacement (phase de quête), rencontre avec un animal substitut de l'être surnaturel, alliance, puis transgression d'un interdit (meurtre et manducation de l'animal), transgression cathartique qui permet la création d'un ordre nouveau. Ainsi, le clan se trouve lié de manière presque ontologique à son culte lequel permet de réaffirmer l'origine commune d'un certain nombre d'individus, de lignages, origine qui concerne leur statut d'être humain et de membre d'un lignage.

${ }^{13} \mathrm{Cela}$ peut sembler contradictoire par rapport au récit que nous avons cité où la mise à mort du lépreux est antérieure à la rencontre avec didiro. Cependant, le motif du "meurtre" et de la "résurrection" soit de dofini sous la forme du soleil (wii), soit de doo (ici de didiro) - apparâtt très souvent dans les mythes sans qu'il soit tenu compte de la chronologie des événements de l'histoire racontée. 


\section{Une entité double}

La dénomination didiro désigne donc à la fois un animal (le poisson), un objet (l'autel de peaux de chiens) et une entité surnaturelle (le didiro, avatar de doo). didiro est représenté graphiquement de deux manières : sous l'aspect du poisson, tenant un rhombe dans sa gueule, et comme paquet oblong ayant deux extrémités différentes. didiro apparait aussi sous la forme de points, les mêmes que ceux qui ornent la peau du poisson sur la "canne de doo", doo buni, outil rituel tenu à la main par le masque de feuilles, masque du culte de doo (voir dessin). Que ce soit dans le mythe ou dans les pratiques cultuelles, la relation à doo est, nous le voyons, très marquée.

didiro, en tant que figure du monde animal, n'est pas à prendre comme entité biologique : seule est retenue comme caractéristique spécifique à l'espèce la faculté de décharger des ondes électriques, faculté amplifiée par les besoins de l'interprétation mythique.

Comment se situe didiro-objet par rapport au mythe? Deux lieux se répondent : la mare, située à une certaine distance du village, et la "maison des vieux pères", ou ancêtres, mieba zii, située au coeur du village. Dans ces deux lieux se trouvent didiro-poisson d'une part, et didiro-objet de l'autre. Dans l'ordre conceptuel, I'un et l'autre sont, nous l'avons vu, la manifestation d'une même entité, l'une vivante et toujours présente dans l'espace sauvage, l'autre, "vivante" d'une autre manière dans l'espace du village. Dans le premier cas, ce n'est pas tant l'animal que le lieu naturel dans lequel il est qui importe; dans le second cas, à l'inverse, c'est la structure de l'objet qui nous intéresse et non pas l'environnement qui ne le distingue pas d'autres objets cultuels situés au même endroit. Les éléments contenus dans le didiro appartiennent aux trois univers, animal, végétal, minéral, et ont été choisis en fonction des figures principales du récit de fondation du clan forgeron.

Entre les deux entités didiro qui coexistent, l'une en milieu naturel, l'autre à l'intérieur du village, il n'y a pas opposition 
mais continuité, un sacrifice au didiro-poisson étant du même ordre qu'un sacrifice au didiro-objet 14 . L'espace de la mare est le lieu de la mise en scène de I'épisode mythique : chaque visite au didiro de l'eau semble être une reconstitution de l'événement passé. Par contre, le didiro du village se situe bien, lui, dans un temps non plus mythique, puisqu'il est l'objet de révélation, mais historique. Le didiro $d u$ village est un artefact, une fabrication humaine. Il est la marque visuelle, plastique, du contrat initial, celle des transformations opérées sur les récepteurs du don divin, celle de la transformation de l'instance surnaturelle elle-même, par le meurtre rituel originel: le fait qu'il y ait, contenu dans le didiroobjet, un fragment de didiro-poisson, est bien la reconnaissance de ce meurtre.

\section{L'identité cachée}

didiro, comme de nombreux objets-fétiches, se caractérise par sa fabrication composite, et par la référence à une première rencontre avec un être surnaturel; il met en relation les deux espaces que nous avons cités, la mare, espace commémoratif du lieu originel mythique, et la maison du village. Si nous regardons maintenant la structure de l'objet, nous voyons qu'il comprend lui aussi deux parties (dans sa composition interne comme dans ses univers de référence externes): on distingue un noyau, - l'aggloméré d'éléments qui se rapportent à l'histoire des didiro et différent selon les clans -, et une enveloppe, les peaux de chiens. Le noyau est la marque matérielle de la "première rencontre" et de la fondation du clan; c'est la marque d'un événement unique. L'enveloppe, par contre,

14 Notre connaissance sur le partage des sacrifices entre mare et village est encore incomplète: les récits de sacrifice à didiro, au lieu de la mare, se rapportent toujours au passé. Il s'agit alors de consulter le génie lui-même et la peau du chien sacrifié serait rapportée au village et ajoutée aux précédentes. Le didiro du village $n^{\prime}$ 'est pas en effet la manifestation du gérile même, mais l'objet qu'il a donné aux hommes. Aller sacrifier au marigot serait alors s'adresser directement au génie sans passer par l'intermédiaire de l'objet. 
est constituée d'événements multiples, puisque chaque peau de chien ajoutée renvoie à un événement grave ou important concernant les lignages. Chaque nouvel acte d'enveloppement, et I'on imagine qu'il en est de même pour tous les objets nazii de ce type, peut être considéré comme un acte de remémoration de la fondation du groupe. Nous rejoignons ici la symbolique du paquet ou du noeud, celle que l'on retrouve dans des amulettes comme le tafo bambara qui est un fil de coton noué. L'état de "noué" présuppose l'action, l'événement de nouer, et est la manifestation visible, la mise en mémoire de cette action, sa fixation symbolique en un lieu.

La bipartition de la composition du didiro peut être transcrite ainsi, en tenant compte du rapport d'inclusion du noyau dans l'enveloppe :

$$
\frac{\text { unique }}{\text { "noyau" }} \quad \frac{\text { multiple }}{\text { "enveloppe de peaux" }}
$$

Cette bipartition s'affine si l'on postule que le "noyau" a une fonction de représentation du groupe humain socialement constitué, les forgerons didiro: les éléments naturels formant le noyau de l'objet apparaissent comme les substituts du clan didiro. Ils constituent en effet l'ensemble synthétique de ce qui est essentiel pour que le clan, ou le lignage, se reconnaisse comme tel, puisqu'ils sont choisis parmi les figures importantes du récit mythique.

Il peut être alors avancé que le groupe se pense comme singulier, unique - chaque noyau différant des autres noyaux -, inclus dans un ensemble plus grand, la communauté villageoise à travers l'enveloppe de peaux de facture identique pour tous. "Emballer", ou ajouter une peau de chien à celles qui existent déjà autour du noyau, c'est non seulement accomplir le même geste que celui effectué par les autres groupes mais c'est aussi maintenir le secret de l'origine, de la fondation du clan (le noyau étant toujours invisible et le secret de sa composition sévèrement gardé). L'enveloppement permet de conserver le noyau dans son intégralité (il n'est pas vu et les éléments qui le composent demeurent intacts) : veiller à la permanence du noyau, c'est, symboliquement, veiller à la permanence 
du groupe dans son identité.

Le tableau suivant permet de formaliser comment sont pensées, à travers didiro, les relations du clan à l'ensemble de la communauté, le noyau étant, de par sa constitution, particulier pour chaque clan propriétaire et l'enveloppe de peaux étant quant à elle d'un usage propre à une communauté élargie.

didiro

\begin{tabular}{|c|c|}
\hline $\begin{array}{l}\text { "noyau" } \\
\text { unique }\end{array}$ & $\begin{array}{l}\text { "enveloppe de peaux" } \\
\text { multiple }\end{array}$ \\
\hline $\begin{array}{l}\text { stable } \\
\text { identité }\end{array}$ & $\begin{array}{l}\text { variable } \\
\text { altérité }\end{array}$ \\
\hline
\end{tabular}

La morphologie de l'objet est variable puisque la partie "enveloppe de peaux" est continuellement en transformation par ajout d'éléments au fur et à mesure des sacrifices - variabilité qui n'est pas totale mais partielle, étant contrainte par un programme d'addition d'éléments toujours semblables, les peaux de chiens.

\section{L'identité énoncée}

La structure double de didiro renvoie également à une complexité de l'énonciation rituelle. Nous pouvons penser en effet qu'à chaque fois qu'il y a adjonction d'une nouvelle peau, il y a un acte d'énonciation qui corresponà à l'acte rituel du sacrifice suivi du geste d'enveloppement. L'enveloppement apparait comme la marque, entre autres possibles, d'un acte d'énonciation, celui du groupe forgeron qui se reconnât comme un "je" (en réalité un "nous", qui n'est qu'un "je" dilaté, si l'on reprend les termes de Benveniste), par rapport aux autres membres de la communauté. Mais ce "je" de l'énonciation ne renvoie pas: à la construction de l'énoncé correspondant qui serait celle du noyau. Celui-ci est en effet conçu et fabriqué une fois pour toutes (dans le mythe, il est donné tel quel comme objet entier par le génie-poisson au premier ancêtre). L'acte d'énonciation 
se fait par contre par un geste, qui ne concerne pas seulement l'ensemble des lignages d'un même clan, mais la communauté toute entière, - puisque d'autres clans procèdent au même rituel lorsqu'ils emballent leurs propres objets -, et qui, dans ce sens, est un geste mimétique. Le sujet de l'énonciation, le groupe clanique, se dédouble : par l'acte d'enveloppement, il s'affirme à la fois comme sujet autonome, individuel, ayant son identité propre, manifestée par ce noyau qui est l'image synthétique de son histoire particulière, et comme sujet participant d'un groupe plus large, l'ensemble de la communauté.

L'acte d'enveloppement fait passer didiro d'un état à un autre (une peau + une autre) : à chaque nouvel enveloppement, il y a transformation d'objet. Mais didiro est en relation analogique avec l'histoire du groupe, il est son identité matérialisée. didiro modifié (il y a une peau en plus) est comme le groupe qui, une fois le sacrifice et l'adjonction d'une peau accomplis, a réaffirmé son identité et "mémorisé" un événement supplémentaire. En conclusion, l'acte d'énonciation, l'enveloppement, permet la conjonction du clan avec la valeur d'identité qui lui est nécessaire pour se reconnâtre en tant qu'unité spécifique à l'intérieur d'un ensemble plus vaste.

\section{Un objet historique}

Du didiro, qui est lié à une certaine représentation de l'identité du groupe, nous avions dit au début de notre analyse qu'il était situé dans un espace caractérisé comme étant près du corps. Caché effectivement dans l'espace de la maison des ancêtres, il est aussi pensé comme un corps. Dans l'article de J. Capron (1957: 58), on retrouve bien nommées les deux parties essentielles, le noyau, "les racines de l'autel", et l'enveloppe, "l'habit de nadâ" (la fabrication se disant "enroulement de nadû", ho nadúbiro). D'autre part, les forgerons didiro donnent les mêmes précisions que les informateurs de cet auteur : l'objet a une tête, située en l'air, et une queue, proche du sol. Cette dernière, constituée de l'ensemble 
des appendices des animaux sacrifiés, doit demeurer toujours "brillante" : si didiro peut être considéré comme un objet organique, en ce sens qu'il est fait de tissus vivants à l'origine (peaux, os du squelette...), il est aussi comme un organisme vivant.

Bien que le noyau constitue une sorte de synthèse des éléments transformés, provenant de la mare, il est cependant dans un état sec. Or, le vivant se caractérise par I'humide. I'humide est bien sûr le trait déterminant du milieu aquatique, la mare et ses alentours, où vit didiro-poisson, mais cette humidité-là n'est pas essentielle, dans la mesure où tous les autels de type nazii ne sont pas forcément composés d'un noyau dont les éléments ont été prélevés en milieu humide. C'est de l'humidité de la peau du chien sacrifié qu'il s'agit, celle qui périodiquement vient se superposer aux autres. A l'humidité de la nouvelle peau vient s'ajouter le "brillant" des queues, elles aussi renouvelées. "Vivant", le didiro l'est, - on le nourrit de sang et d'humidité, il doit être toujours brillant, etc -, mais il l'est aussi parce qu'il demeure un objet non-fini, en transformation, pris dans un devenir, celui de l'ajout progressif des peaux.

Revenons à ce que nous avions dit précédemment : didiro, bipartite, renvoie par son noyau à un événement unique, la première rencontre et la fondation du clan, et par son enveloppe à des événements multiples, manifestés par les peaux. D'un point de vue temporel, la bipartition semble pouvoir s'articuler en fonction d'un temps rétrospectif, représenté par le "noyau" et d'un temps prospectif, manifesté par les "peaux". Le temps rétrospectif est celui qui se réfère au récit mythique de la première rencontre, au "passé de l'expérience" (Benveniste, 1974: 76) et le temps prospectif à celui du groupe en histoire. Mais si le temps rétrospectif part d'un "moment axial", d'un "point zéro" (ibid.: 71), la rencontre avec didiro-poisson, le temps prospectif, celui de l'objet historicisé, ne peut être conçu dans une dimension chronologique que par la mise en actes qu'opère le rituel. C'est bien le rituel qui, par l'adjonction des peaux, positionne l'objet dans cette dimension du devenir. Concevoir l'objet en diachronie, c'est le projeter dans une dynamique où se succèdent des événements, sans réversibilité 
possible. Chacun de ces événements est unique : en effet, l'adjonction d'une peau de chien correspond à un événement particulier concernant chaque lignage didiro. Nous pourrions alors dire que la taille de l'objet est proportionnelle à la somme des événements survenus au sein de ce lignage et ayant nécessité un sacrifice de chien : l'objet se dilate, grossit en fonction de ceux-ci. L'histoire du groupe est mise en objet.

Si ces événements sont de l'ordre de la contingence (à part les offrandes périodiques qui ne sont nécessitées par aucun événement particulier), puisqu'ils représentent ce qu'il advient au groupe, l'énonciation sacrificielle et rituelle qui en découle est nécessaire au renouvellement de l'affirmation de l'identité du lignage. didiro apparâ̂t lié à l'histoire événementielle, donc transformationnelle, du groupe : cela ne veut pas dire que la forme de l'objet en soit totalement dépendante, mais plus simplement, comme nous l'avons dit en introduction, qu'elle évolue en parallèle. Un geste peut être considéré comme égal à une trace d'événement qui, tout en étant unique, se perd dans l'uniformité de la structure feuilletée des peaux mises en paquet. Cette suite d'événements qui engendrent l'objet, ou l'ont engendré, le placent dans une durée temporelle, celle du procès de fabrication, partant du "point zéro" de la première rencontre, et tendant vers le nonfini:

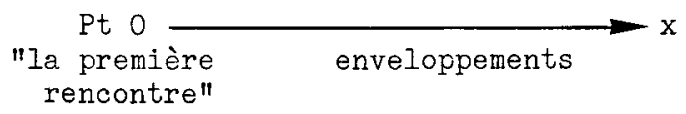

Compris entre le point zéro et le non-fini d'un temps $x$, chaque enveloppement nécessite un événement pour être matérialisé par une trace (une peau). L'objet ne peut alors être considéré comme fini que lorsque le groupe lui-même n'est plus en mesure de continuer à le construire par de nouveaux ajouts de peaux (extinction ou dispersion des lignages, etc.). Si chaque trace (peau de chien) est celle d'un événement passé, - celui qui a occasionné le sacrifice -, il en résulte bien que didiro n'est conçu comme "vivant", opérationnel, que dans la mesure où il est toujours en transformation. 
A l'image du groupe humain vivant, didiro est dans ce qu'on pourrait appeler un état de genèse, de formation continue. Sa forme visuelle, ses contours, sont toujours indéterminés puisque toujours susceptibles d'être modifiés. Il ne peut pas régresser dans sa forme, devenir plus petit, rétrécir : il est pris dans un procès d'accumulation régulière d'éléments identiques. Cette accumulation est un des facteurs qui composent l'esthétique qui lui est propre. Il est considéré comme beau, bon (le terme est le même dans les deux cas, si) lorsqu'il est de taille imposante. Nous retrouvons cela à propos des fétiches à clous du Zaïre, les nkisi, jugés de façon similaire par leurs propriétaires : "Plus il y a de clous, plus le nkisi est beau" (Lehuard, 1980: 250). La somme des clous additionnés correspond là-aussi, comme pour les peaux de chiens, à autant d'actes rituels qui mettent en forme l'objet et l'inscrivent dans une temporalité déterminée. Dans certains cas, la forme anthropomorphe ou zoomorphe qui constitue l'armature centrale de l'objet disparalt sous l'amas de clous et d'instruments métalliques divers qui y sont plantés. Comme pour le didiro, la morphologie est variable - la forme et ses contours demeurent changeants - par nécessité.

didiro participe de la religion de doo : à notre connaissance, il n'est jamais utilisé à des fins magiques, si l'on donne à ce terme le sens de manipulations de "forces" bénéfiques ou maléfiques. Il diffère en cela de nombreux objets appelés fétiches, tels que les nkisi ou les vodoun de la côte. Cependant si l'usage n'est pas le même, la forme, ou plutôt le type de forme (non fini, en transformation), ainsi que la matière (composite, hétérogène) sont semblables. Au cours de cette analyse a été étudié le rôle que tient un objet comme le didiro dans les rituels qui lui sont liés, en fonction d' une organisation sociale et de certaines données mythiques: celles-ci sont projetées symboliquement dans la structure de l'objet (noyau + enveloppe feuilletée) dont rien ne prouve par ailleurs qu'il n'ait été emprunté, à une époque plus ou moins récente, à une ethnie voisine (les forgerons didiro disent qu'il leur a été donné tel quel, 
déjà fabriqué, par didiro-poisson : il n'y a pas, comme pour beaucoup d'autres objets, une histoire mythique de sa fabrication).

L'interprétation de la forme se développerait donc par rapport à ces variables, propres à chaque société ; mais la pensée formelle elle-même est indépendante de ces variables au point de constituer une esthétique de l'objet fétiche, toutes sociétés confondues. Une esthétique qui, par la récurrence de la même logique interne organisant les éléments formels, en vient à produire un style particulier. Ce style peut être conçu comme la manifestation d'un système composé de termes et de relations : l'étude des termes est celle des éléments choisis pour construire l'objet en tenant compte de leurs qualités formelles et matérielles (en dehors du sens qui y est investi), éléments végétaux, animaux, peaux, cordes, clous, etc. Celle des relations a été abordée dans les pages précédentes à travers le principe d'addition d'éléments semblables, les peaux, modifiant à chaque fois l'objet.

Nous avons vu qu'il s'agissait en général d'éléments directement pris à la nature; mais lorsque ceux-ci sont transférés tels quels de leur milieu naturel à l'objet, même s'ils ne changent pas dans leur apparence, - nous pensons, par exemple, à des objets-fétiches comportant des coquillages disposés en couronne (Luba du Kasaï)-, ils changent dans leur fonction puisqu'ils ne sont plus intégrés au même système de relations. Le coquillage n'est plus la coquille de l'animal perçu dans l'environnement qui lui est propre mais appartient dès lors à un ensemble d'éléments autres, ceux choisis par l'homme pour construire son objet, organisation signifiante comme celle du monde naturel mais différente : si chaque élément a sa spécificité de forme et de matière, celle-ci doit être appréhendée dans l'ensemble des relations qui organisent l'objet lui-même.

L'iconographie se rapportant à un style "fétiche" pourrait s'articuler sur ce principe : variation du sens pour une même forme. Le style "fétiche" appartient en effet à une famille de formes qui dépasse le contexte africain : s'opposant à celui des oeuvres plastiques achevées, montrées dans leur état définitif, il est présent dans les oeuvres d'art européennes et contemporaines. Nous pensons en particulier à certaines pièces de Picasso, obtenues par adjonction 
d'éléments non transformés - cuiller, cordes, tasseaux de bois et qui font sculpture. S'il s'agit avant tout d'une parenté formelle, l'idée d'une transformation inaccomplie demeure. En effet, le bois du corps d'une statuette n'est plus le bois de l'arbre; mais, dans le cas de l'objet-f'étiche, le matériau prélevé à l'environnement naturel (peaux, ossements, végétaux) ou à celui des objets manufacturés (clous, cordes, tissus) est employé tel quel pour construire l'objet. L'objet-fétiche est complexe puisque constitué de la somme d'éléments hétérogènes; tout fétiche doit être cependant considéré comme un objet unique, parce qu'il est le résultat d'une combinaison en principe originale de matériaux ayant chacun leur particularité. Chaque matériau se réfère en effet à une histoire qui justifie sa présence dans l'objet, mais possède aussi des qualités matérielles et formelles qui jouent en différence et en contraste avec celles des autres matériaux. Chaque élément demeure autonome : une peau est une peau, un clou est un clou. La juxtaposition d'une peau d'animal avec un clou fait contraste car les univers de forme et de matière invoqués sont étrangers l'un à l'autre. La mise en évidence de ces contrastes, dont le spectateur ressent très fortement la présence, peut-être plus encore que la structure d'accumulation ou d'empaquetage que nous avons étudiée, est une des données fondamentales de l'esthétique fétiche.

C'est dans ce sens qu'il nous semble que la dénomination "fétiche" peut être utile : non plus pour désigner certains objets en fonction de critères symboliques ou religieux, mais en fonction de critères plastiques.

Que ce soit le didiro - où l'on sait, plus qu'on ne le voit, ce qu'est l'objet - ou les fétiches à clous ou à miroir de l'Afrique centrale (populations Kongo, Luba, Lumbo, etc.), d'un point de vue esthétique, nous avons affaire au même type d'objet. Mais, l'iconographie à notre disposition pour une recherche esthétique à venir sur l'objet-fétiche est malheureusement très pauvre, n'ayant retenu que ceux où apparaît une figuration anthropomorphe ou zoomorphe qui renvoie à une vision esthétique classique privilégiant ces représentations.

Michèle Coquet

U.R.L. 7 - C.N.R.S. 


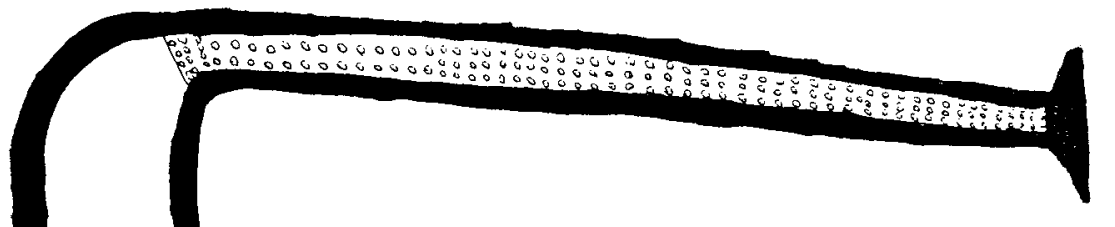

doo buni, "canne de doo", instrument rituel du masque de feuilles. Le manche est recouvert d'une peau d'iguane dont les grains sont pour les didiro 1 'équivalent des points qui ornent 1 a peau du poisson.

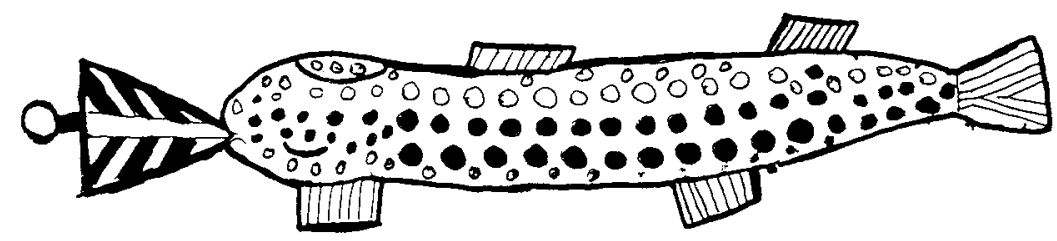

didiro-poisson vomissant le rhombe

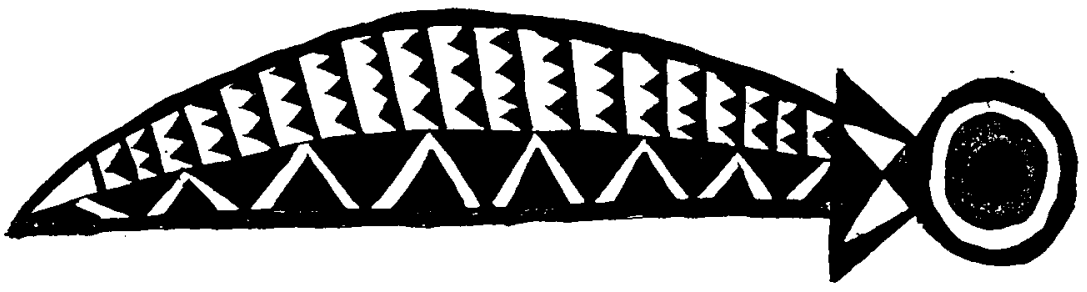

didiro-objet tel qu'il est dans la maison des ancêtres.

Trois représentations de didiro dessinées par Luoho Didido, chef des forgerons de Houndé. 


\section{Bibliographie}

Benveniste E.

1974 Problèmes de linguistique générale 2, Gallimard, Paris.

Boas F.

1955 Primitive Art, Dora Publications, Inc., N.Y.

Capron $J$.

1957 "Quelques notes sur la société do chez les populations bwa du cercle de San", Journal de la Société des Africanistes, XXVII.

1978 "Sur deux noms personnels bwa", Systèmes de signes, Hermann, Paris.

Coquet $\mathrm{H}$.

1984 "Une représentation plastique du temps", Systèmes de Pensée en Afrique Noire, 7.

Evans-Pritchard E.E.

1971 La religion des primitifs, Petite bibliothèque Payot, Paris.

Guébhard P.

1911 "Notes contributives à I'étude de la religion, des moeurs et des coutumes des Bobo du cercle de Koury (Soudan français)", Revue d'Ethnographie et de Sociologie, 2, 5/6.

Heusch de L.

1974 "Introduction à une ritologie générale", L'unité de l'homme, 3, Points, Seuil, Paris.

Labouret $\mathrm{H}$.

1927 "Quelques aspects de la magie noire africaine, amulettes et talismans du Soudan français", Bulletin du Comité d'études historiques et scientifiques de I'A.0.F., X, 3-4.

Laude $J$.

1979 Les arts d'Afrique noire, Chêne, Paris.

Lehuard $R$.

1980 Fétiches à clous de Bas-Zaïre, Ed. Arts d'Afrique noire.

Le Moal G.

1980 Les Bobo, Nature et fonction des masques, ORSTOM, Paris.

1983 "Code sacrificiel et catégories de pensée chez les Bobo", Systèmes de pensée en Afrique noire, 6 .

Ratton C.

1951 "L'or fétiche", Présence africaine, 10-11, Paris. 


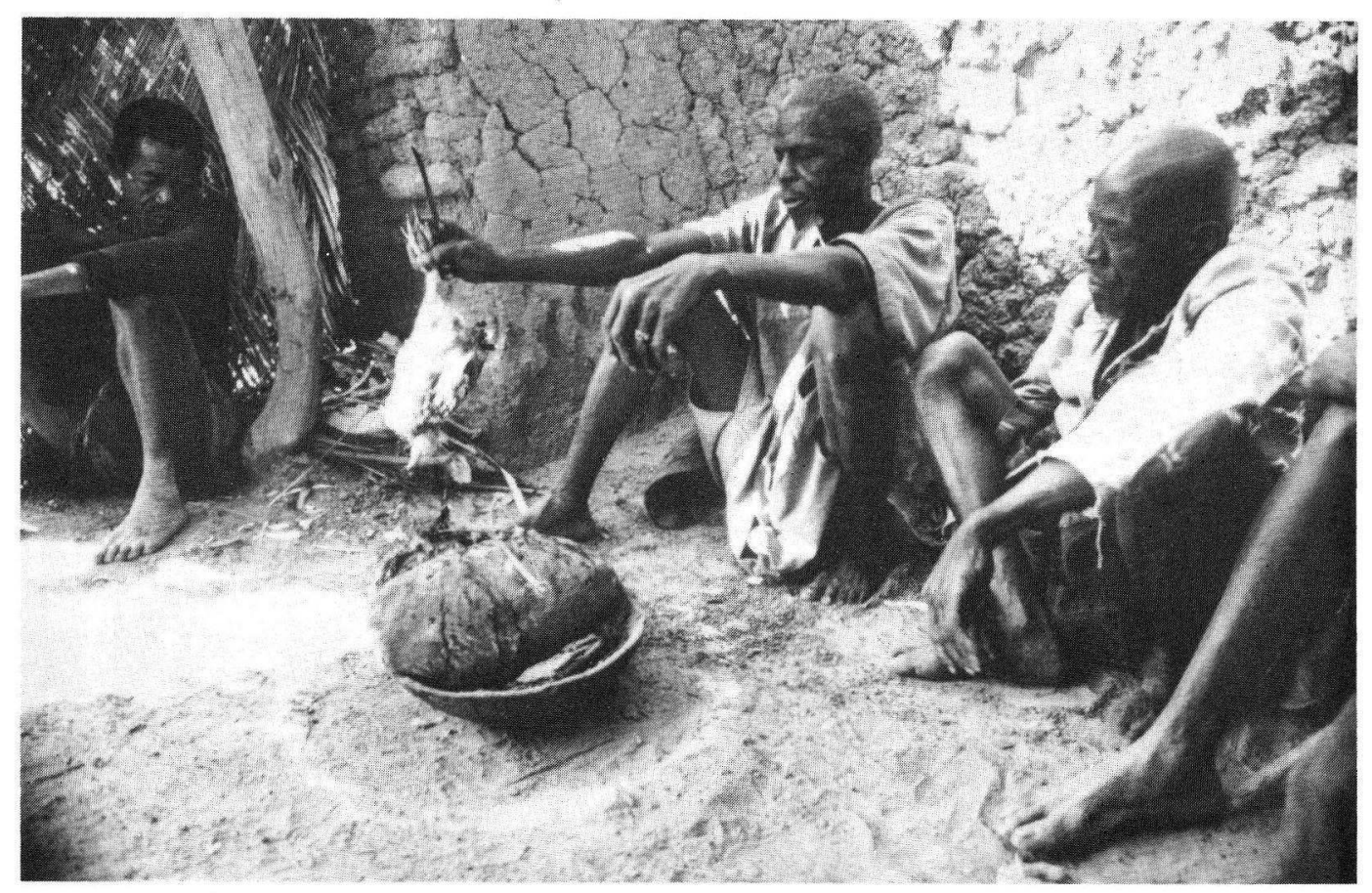

Objet wahu, de type nazii, clan Coulibali, village de Bouéré, utilisé ici pour une ordalie concernant un clan d'un autre village, ce qui est un cas exceptionnel. 